\title{
Abdominal obesity phenotypes outcomes: protocol for a systematic review
}

soraya doustmohamdian ( $\square$ sorayadoust@yahoo.com )

Semnan University

farhad hosseinpanah

Shaheed Beheshti University of Medical Sciences

marjan momeni

Semnan University

\section{Protocol}

Keywords: Abdominal obesity, cardiovascular disease, all-cause mortality

Posted Date: May 18th, 2020

DOl: https://doi.org/10.21203/rs.3.rs-27658/v1

License: (c) (1) This work is licensed under a Creative Commons Attribution 4.0 International License. Read Full License 


\section{Abstract}

Background: The prevalence of obesity is increasing worldwide. Obesity is associated with severe health effects. Abdominal obesity has a strong association with metabolic dysfunction, diabetes mellitus type 2 (DM2), dyslipidemia, hypertension, and coronary artery events.

Methods: We will search PubMed/MEDLINE, EMBASE, Web of Science, Cochrane Library, and ProQuest (from inception onwards). Additional studies will be identified through manual searching of reference lists. Quantitative studies evaluating abdominal obesity phenotype outcomes in adults will be included. Primary results will be assaying abdominal obesity phenotype results, including DM2 incidence, cardiovascular disease risk, and all-cause mortality. Two reviewers will independently screen full-text articles and abstract data. Potential conflicts will be resolved through discussion. The study methodological quality (or bias) will be appraised using appropriate tools. If feasible, we will conduct a random-effects meta-analysis. The two researchers will also assess the quality of the articles independently based on CASP.

Discussion: This systematic review will summarize evidence regarding the association between abdominal obesity phenotypes with DM2,cardiovascular disease and all cause mortality. The results of this review will provide a useful reference for the importance of abdominal obesity on metabolic dysfunction and mortality

\section{Background}

The prevalence of obesity is rising across the world. In the United States, the rate of obesity in adults was about $35.7 \%$ in 2010 (1). Obesity is associated with severe health effects such as hypertension, dyslipidemia, insulin resistance, type 2 diabetes, and cardiovascular disease (2).

Abdominal obesity has a stronger association with metabolic dysfunction than generalized obesity. Some studies have shown that abdominal obesity is an independent risk factor for diabetes mellitus type 2 (DM2), dyslipidemia, hypertension, and coronary artery events. The risk of cardiovascular and all-cause mortality increases in abdominal obese populations in parallel with waist circumference (WC) (3-5).

A subgroup of people with central obesity has been identified without typical metabolic disorders associated with obesity. Metabolically healthy abdominal obesity (MHAO) phenotype has been previously defined as a subgroup of abdominal obese individuals who do not have insulin resistance, dyslipidemia, or hypertension (6). Some studies indicate that $23.5 \%$ of abdominal obese cases can be categorized as $\operatorname{MHAO}(7,8)$.

\section{Objectives}

The purpose of this review is to evaluate the MHAO phenotype in the context of type $2 \mathrm{DM}$ incidence, cardiovascular disease risk, and all-cause mortality. 


\section{Methods}

Protocol: The protocol of the systematic review was drafted and uploaded to the PROSPERO website. The protocol code was issued by PROSPERO (CRD42019111056) and will be reported based on the reporting guidance provided in the Preferred Reporting Items for Systematic Reviews and Meta-Analyses Protocols (PRISMA P) statement (9). The methods and results will also be reported according to the Preferred Reporting Items for Systematic Reviews and Meta-Analyses (PRISMA) statement (10).

\section{Eligibility Criteria for considering studies for this review}

Types of studies: Human quantitative studies (e.g., case-control studies and cohort studies) evaluating the association between abdominal obesity phenotype outcomes in adults will be included.

Types of participants: We will assess all studies targeting adults (>20 years old) of abdominal obese phenotypes and evaluating the association of different abdominal obesity phenotypes (in compared with healthy non-abdominal obese phenotype individuals as the reference group) with DM2 incidence, cardiovascular disease risk, and all-cause mortality.

We will consider at least four groups as exposure:

(i) Metabolically healthy abdominal obese (abdominal obese without metabolic syndrome),

(ii) Metabolically healthy non-abdominal obese (non-abdominal obese without metabolic syndrome),

(iii) Metabolically unhealthy abdominal obese (abdominal obese with metabolic syndrome),

(iv) Metabolically unhealthy non-abdominal obese (non-abdominal obese with metabolic syndrome).

Types of result: We will assess all studies with their primary results on DM2 incidence, cardiovascular disease (fetal or non-fetal) risk, or all-cause mortality. We will report risks as an incidence rate, a relative risk, or odds.

\section{Search methods for identification of studies}

\section{Electronic searches}

To access studies conducted on abdominal obesity phenotypes and their outcomes (risk of type 2 DM, cardiovascular disease, and all-cause mortality), we will search PubMed/MEDLINE, EMBASE, Web of Science, Cochrane Library, and ProQuest (from inception onwards). Additional studies will be identified through manual searching of reference lists. The search will include a broad range of terms and keywords, including "central adiposity", "abdominal obesity", "obesity, abdominal", "abdominal fat", "diabetes mellitus type 2", "cardiovascular diseases", mortality, and "metabolically healthy". To access all 
relevant articles, the reference list of review articles and meta-analyses (backward searching), cited articles (forward-searching), and papers introduced as "related articles" will be checked. Searches resulting in peer-reviewed articles, letters, abstracts, or editorials will be excluded.

\section{Data collection and analysis}

\section{Selection of studies}

All studies obtained from different sources will be transferred to Endnote, and duplicates will be systematically removed so that a merged library can be created. Two reviewers will independently screen titles and abstracts according to pre defined inclusion and exclusion criteria checklist to identify potential studies for reviewing and exclude unrelated articles. In case of disagreement between the two reviewers, the final decision and judgment for including the study will be made based on the inclusion criteria with the opinion of a third person.

Full texts will be read by the two individuals separately, and final decisions will be made based on the inclusion criteria checklist.

As criteria to define metabolically healthy or abdominal obesity may vary in different studies, we will accept the definitions of them provided by authors in each study. If necessary and possible, we will contact the authors of studies to resolve any ambiguities. A third reviewer will decide any discrepancies in the selection of studies for inclusion in the review. All three reviewers will verify the final list of included studies in the review, and a PRISMA diagram will be used to show steps for inclusion of selected articles.

In this study, the search strategy and the screening and selection of the data will be based on the Preferred Reporting Items for Systematic Reviews and Meta-Analyses (PRISMA) guidelines.

\section{Data extraction}

An extraction form will be designed to collect information from each study that will include the following:

1- Study characteristics: title, study design, year of publication, journal, author and period of follow up,

2- Methods: eligibility of study based on inclusion criteria, purpose of study, method of data collection, and sampling methods,

3- Participant characteristics: sample size, age (e.g., mean with standard deviation, range, etc.), gender, and definition of abdominal obesity and metabolic syndrome,

4- Results: primary results, including DM2 incidence, cardiovascular disease risk, and all-cause mortality. 


\section{Assessment of risk of bias in included studies}

\section{Appraisal of study quality}

Two independent investigators will review study titles and abstracts, and studies satisfying the inclusion criteria will be retrieved for full-text evaluation. A third investigator will resolve disagreements. Two independent project collaborators will assess full texts of eligible articles. The two researchers will also assess the quality of articles independently based on the Critical Appraisal Skills Programme (CASP) 2018. The CASP checklist includes 10 questions to help us make sense of qualitative research. The instrument assesses result validity, research design, recruitment strategy, sample size, clear reporting of finding, data collection, ethical issues, and statistical analysis using a simple "yes", "no", or "can't tell". The results of the assessment will be shown in a table format.

All three reviewers will resolve any differences in the quality assessment of articles by discussion.

\section{Data synthesis}

The information for each study (i.e., study characteristics, participants, outcomes, and findings) will be used to build evidence tables of an overall description of the included studies.

\section{Additional analyses}

We will meta-analyze data from comparable studies if at least two studies are available. If studies are sufficient, and all data are available, sources of heterogeneity of studies will be further investigated by subgroup or meta-regression analysis. We will use the Cochran $\mathrm{Q}$ test to evaluate heterogeneity between studies, and consider a threshold $P$ value less than 0.05 as statistically significant. In the presence of heterogeneity, if possible, subgroup analyses based on age, sex, quality of article (low, moderate, or high risk of bias), length of follow up, and clinical outcome will be performed. We will also plan to evaluate the heterogeneity magnitude between studies using the $I^{2}$ testing. If quantitative synthesis is not appropriate, we will use a summary table to describe definitions of metabolically healthy and abdominal obesity, sample size, outcome of interest, and duration of follow up. The findings of articles will be discussed, and the conclusion will depend on the power and strength of each study.

\section{Discussion}

This systematic review and meta-analysis will be the first of its kind in explaining the relationship between abdominal obesity phenotypes, mortality, and morbidity.

It appears that a certain proportion of abdominal obese individuals have a normal metabolic profile. It is unclear whether this group (MHAO) express a lower risk of all-cause mortality, CVD, or DM2 compared to 
"metabolically unhealthy" abdominal obese. Although individuals with MHO phenotype appear to be less at risk for cardiovascular events or mortality than those with MONW phenotype (11-13), abdominal obesity can be associated with increased cardiac and overall mortality, independent of generalized obesity, based on BMI $(14,15)$. Lower WC in MHO phenotype, despite higher BMI, may justify a reduction in mortality or CVD in this group (16). Therefore, abdominal obesity may be a more important factor than BMI for CVD or mortality. This systematic review will summarize evidence regarding the association between abdominal obesity phenotypes with DM2, cardiovascular disease and all cause mortality

The results of this review will provide a useful reference for the effect of abdominal obesity on metabolic dysfunction and cardiovascular or all-cause mortality.

\section{Abbreviations}

MHAO: Metabolically healthy abdominal obese, MHNAO: Metabolically healthy non-abdominal obese MUAO: Metabolically unhealthy abdominal obese, MUNAO: Metabolically unhealthy non-abdominal obese, MHO:: Metabolically healthy obese, MONW: Metabolically Obese Normal Weight, PRISMA-P: Preferred Reporting Items for Systematic Review and Meta-Analysis Statement-Protocol Extension, WC: waist circumference, BMI: body mass index, CVD: cardiovascular disease, DM2: diabetes mellitus type 2, CASP: Critical Appraisal Skills Programme

\section{Declarations}

The protocol does not represent an amendment of a previously completed or published protocol. protocol is not for an update of a previous systematic review.

\section{Ethics approval and consent to participate}

Not applicable

\section{Funding}

This review do not have any sponsor.

\section{Consent for publication}

Not applicable

\section{Availability of data and materials}

Data sharing is not applicable to this article as no datasets were generated or analyzed during the current study.

\section{Competing interests}


Authors declare no competing interests

\section{Authors' contributions}

SD and FH designed the study. The search strategy was conducted under the supervision of SD and MM. SD prepared the initial protocol, and revised by FH and MM. All authors read the final protocol and guarantee that the results will be published as a review article and if possible, meta-analysis.

\section{References}

1. Ogden $\mathrm{CL}$, Carroll MD, Kit BK, Flegal KM. Prevalence of childhood and adult obesity in the United States, 2011-2012. Jama. 2014;311(8):806-14.

2. Reilly JJ, Methven E, McDowell ZC, Hacking B, Alexander D, Stewart L, et al. Health consequences of obesity. Archives of disease in childhood. 2003;88(9):748-52.

3. Larsson B, Svärdsudd K, Welin L, Wilhelmsen L, Björntorp P, Tibblin G. Abdominal adipose tissue distribution, obesity, and risk of cardiovascular disease and death: 13 year follow up of participants in the study of men born in 1913. Br Med J (Clin Res Ed). 1984;288(6428):1401-4.

4. Ducimetiere P, Richard J, Cambien F. The pattern of subcutaneous fat distribution in middle-aged men and the risk of coronary heart disease: the Paris Prospective Study. International journal of obesity. 1986;10(3):229-40.

5. Dagenais GR, Yi Q, Mann JF, Bosch J, Pogue J, Yusuf S, et al. Prognostic impact of body weight and abdominal obesity in women and men with cardiovascular disease. American heart journal. 2005;149(1):54-60.

6. Blüher M. The distinction of metabolically 'healthy'from 'unhealthy'obese individuals. Current opinion in lipidology. 2010;21(1):38-43.

7. Doustmohamadian S, Serahati S, Barzin M, Keihani S, Azizi F, Hosseinpanah F. Risk of all-cause mortality in abdominal obesity phenotypes: Tehran Lipid and Glucose Study. Nutrition, Metabolism and Cardiovascular Diseases. 2017;27(3):241-8.

8. Keihani S, Hosseinpanah F, Barzin M, Serahati S, Doustmohamadian S, Azizi F. Abdominal obesity phenotypes and risk of cardiovascular disease in a decade of follow-up: The Tehran Lipid and Glucose Study. Atherosclerosis. 2015;238(2):256-63.

9. Moher D, Liberati A, Tetzlaff J, Altman DG, Altman D, Antes G, et al. Preferred reporting items for systematic reviews and meta-analyses: the PRISMA statement (Chinese edition). Journal of Chinese Integrative Medicine. 2009;7(9):889-96.

10. Krause $\mathrm{C}$, Sommerhalder K, Beer-Borst S, Abel T. Just a subtle difference? Findings from a systematic review on definitions of nutrition literacy and food literacy. Health promotion international. 2018;33(3):378-89.

11. Hosseinpanah F, Barzin M, Sheikholeslami F, Azizi F. Effect of different obesity phenotypes on cardiovascular events in Tehran Lipid and Glucose Study (TLGS). The American journal of 
cardiology. 2011;107(3):412-6.

12. Meigs JB, Wilson PW, Fox CS, Vasan RS, Nathan DM, Sullivan LM, et al. Body mass index, metabolic syndrome, and risk of type 2 diabetes or cardiovascular disease. The Journal of Clinical Endocrinology \& Metabolism. 2006;91(8):2906-12.

13. Romero-Corral A, Somers VK, Sierra-Johnson J, Korenfeld Y, Boarin S, Korinek J, et al. Normal weight obesity: a risk factor for cardiometabolic dysregulation and cardiovascular mortality. European heart journal. 2010;31(6):737-46.

14. Jacobs EJ, Newton CC, Wang Y, Patel AV, McCullough ML, Campbell PT, et al. Waist circumference and all-cause mortality in a large US cohort. Archives of internal medicine. 2010;170(15):1293-301.

15. Leitzmann MF, Moore SC, Koster A, Harris TB, Park Y, Hollenbeck A, et al. Waist circumference as compared with body-mass index in predicting mortality from specific causes. PloS one. 2011;6(4).

16. Calori G, Lattuada G, Piemonti L, Garancini MP, Ragogna F, Villa M, et al. Prevalence, metabolic features, and prognosis of metabolically healthy obese Italian individuals: the Cremona Study. Diabetes care. 2011;34(1):210-5.

\section{Figures}






Figure 1

PRISMA diagram

\section{Supplementary Files}

This is a list of supplementary files associated with this preprint. Click to download.

- CRD42019111056prospero.pdf 
- certificates.pdf

- PICO.doc

- PRISMAPchecklist.docx

- CASP.docx 